\title{
Intraperitoneal chemotherapy for gastric cancer with peritoneal disease: experience from Singapore and Japan
}

\author{
Koji Kono ${ }^{1,2,3}$ - Wei-Peng Yong ${ }^{4} \cdot$ Hirokazu Okayama ${ }^{1} \cdot$ Asim Shabbir $^{2}$ • \\ Tomoyuki Momma $^{1} \cdot$ Shinji Ohki $^{1} \cdot$ Seiichi Takenoshita ${ }^{1} \cdot$ Jimmy So $^{2}$
}

Received: 1 September 2016/Accepted: 10 October 2016/Published online: 20 October 2016

(c) The International Gastric Cancer Association and The Japanese Gastric Cancer Association 2016

\begin{abstract}
Among advanced gastric cancer cases, peritoneal dissemination is a life-threatening mode of metastasis, and any strategy to control peritoneal metastasis will significantly improve treatment outcomes. Since intraperitoneal administration of anticancer drugs can induce an extremely high concentration of drugs in the peritoneal cavity, intraperitoneal chemotherapy would appear to be a reasonable and promising strategy to control the peritoneal dissemination. However, it has been reported in the past that intraperitoneal administration of mitomycin $\mathrm{C}$ or cisplatin resulted in no significant clinical effects against peritoneal metastasis of gastric cancer. In contrast, intraperitoneal paclitaxel is expected to remain inside the peritoneal cavity due to its large molecular weight and fat solubility, leading to a high concentration of the drug in the peritoneal cavity. In fact, promising results in several phase II clinical trials using intraperitoneal paclitaxel have been reported, including a median survival time of 16.2-24.6 months and a 1-year overall survival rate of 69-78 \%. Thereafter, a phase III randomized control study (PHOENIX-GC trial) with intraperitoneal paclitaxel plus systemic S-1 and intravenous paclitaxel in comparison to
\end{abstract}

Koji Kono

kojikono@fmu.ac.jp

1 Department of Organ Regulatory Surgery, Fukushima Medical University, 1 Hikarigaoka, Fukushima 960-1295, Japan

2 Department of Surgery, National University of Singapore, Singapore, Singapore

3 Department of Advanced Cancer Immunotherapy, Fukushima Medical University, Fukushima, Japan

4 Department of Hematology-Oncology, National University of Singapore, Singapore, Singapore systemic S-1 plus cisplatin was conducted in Japan. Moreover, a phase II clinical trial of combination chemotherapy of intraperitoneal paclitaxel with systemic capecitabine plus oxaliplatin is currently ongoing in Singapore. In this review, based on clinical experience from Singapore and Japan, the clinical significance of intraperitoneal chemotherapy for gastric cancer with peritoneal disease is discussed.

Keywords Intraperitoneal chemotherapy · Paclitaxel · Gastric cancer $\cdot$ S- $1 \cdot$ XELOX $\cdot$ Singapore

\section{Introduction}

Gastric cancer is the fourth most common cancer around the world and is the second leading cause of cancer-related death [1]. Among advanced gastric cancer cases, peritoneal dissemination is a life-threatening mode of metastasis, and any strategy to control peritoneal metastasis will significantly improve treatment outcomes. For example, there was a clinical study suggesting that extensive intraoperative peritoneal lavage could reduce the risk of peritoneal recurrence and improve overall survival [2]. However, it is generally accepted that once peritoneal dissemination is established, the disease becomes uncurable and the prognosis of the patient is dismal.

Since intraperitoneal administration of anticancer drugs can induce an extremely high concentration of drugs in the peritoneal cavity, intraperitoneal chemotherapy would appear to be a reasonable and promising strategy for controlling peritoneal dissemination. However, it has been reported in the past that intraperitoneal administration of mitomycin $\mathrm{C}$ (MMC) or cisplatin (CDDP) resulted in no significant clinical effects against peritoneal metastasis of 
gastric cancer [3] due to pharmacokinetic insufficiency or poor sensitivity of the cancer cells to the drug. In contrast, paclitaxel is absorbed slowly through the lymphatic system after intraperitoneal administration due to its large molecular weight and fat solubility, leading to a high concentration of this drug in the peritoneal cavity [4, 5]. Intraperitoneal administration of paclitaxel was developed to control peritoneal metastasis, and its clinical effects have been confirmed by several clinical trials in ovarian cancer with peritoneal metastasis $[4,5]$. Therefore, the application of intraperitoneal paclitaxel to gastric cancer with peritoneal metastasis would appear to be a promising strategy.

In this review, we discuss the clinical significance of intraperitoneal chemotherapy for gastric cancer with peritoneal disease based on clinical experience from Singapore and Japan.

\section{General view of gastric cancer in Singapore}

Cancer is one of the main causes of death in Singapore; it was responsible for $29.3 \%$ of all deaths there in 2009 [6]. Lung, colorectal, and breast cancer were the top causes of cancer burden, and gastric cancer is the fifth most common cancer in men and the seventh most common in women (Singapore Cancer Registry, 2002-2006, [6]). Among the three main ethnic groups in Singapore (Chinese, Malays, and Indians), the Chinese population is most prone to developing gastric cancer, with 1 in 50 Chinese men developing gastric cancer in their lifetime. The incidence of gastric cancer that is at an advanced stage at diagnosis is higher than the corresponding incidences in Japan and Korea: the prevalence of stages III and IV is $57 \%$, whereas that of stages I and II is $42 \%$ [7]. The five-year survival rate of gastric cancer at any stage in Singapore is reported to be $24 \%$, which is comparable to the corresponding global figure (Fig. 1).

In Singapore, the treatment strategy for gastric cancer is based on the Japanese Gastric Treatment Guideline (2014, version 4). For example, D2 lymph node dissection is routinely performed for advanced gastric cancer, and laparoscopically assisted gastrectomy or endoscopic submucosal dissection is regularly considered for early gastric cancer, depending on the diagnosed tumor depth. Although the indication of chemotherapy for gastric cancer in Singapore is similar to the indication of chemotherapy for gastric cancer in Japan, the regimen used is different from the Japanese standard. Since S-1 is not yet widely available in Singapore, a combination of capecitabine with oxaliplatin (XELOX) is currently the most common chemotherapy regimen for patients with advanced gastric cancer. S-1 is an option for adjuvant therapy, since S-1

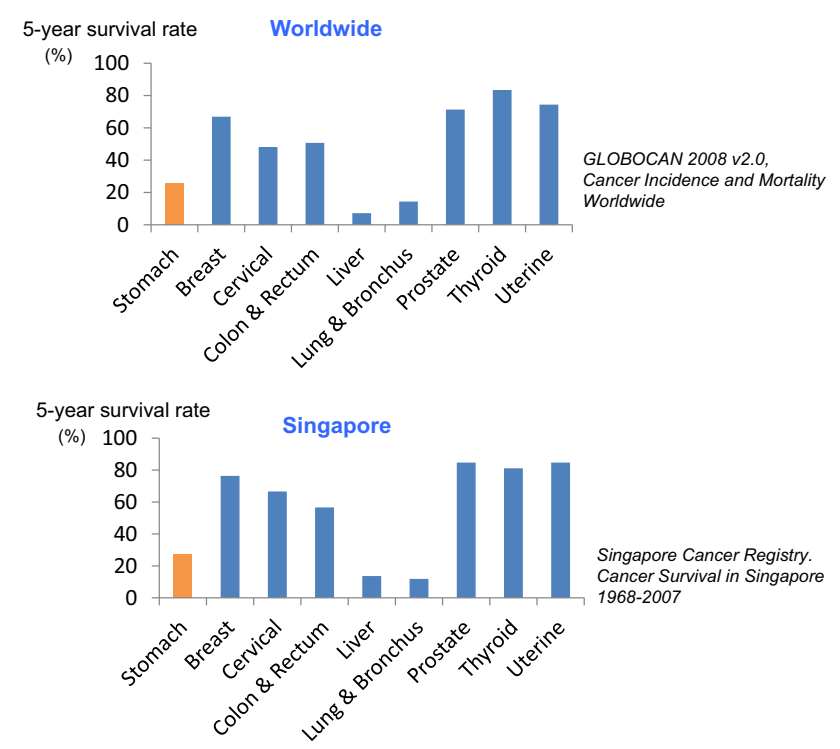

Fig. 1 Five-year survival rates of cancers worldwide and in Singapore

monotherapy is less toxic than the other combination regimen.

In Singapore, there is no special treatment option for gastric cancer with peritoneal dissemination, and it is generally accepted that cases with peritoneal dissemination are inoperable and systemic chemotherapy with XELOX is usually chosen as the treatment. Therefore, a clinical trial with intraperitoneal chemotherapy, a new promising treatment option, is currently being conducted in Singapore, and that trial is discussed later in this review.

\section{General view of gastric cancer with peritoneal dissemination}

The median survival of patients with unresectable or recurrent gastric cancer treated with systemic chemotherapy is about 12 months, and the 1-year overall survival rate is around $50 \%[8,9]$. Among such patients, the median survival of those with peritoneal disease was reported to be dismal: 6-10 months. Although various strategies have been applied to control peritoneal metastasis, including systemic chemotherapy, intraperitoneal chemotherapy, hyperthermia, and aggressive surgery, the clinical benefits of these treatments have proven to be limited $[10,11]$.

Currently, systemic chemotherapy with 5-fluorouracil (5-FU)-based and/or CDDP-based regimens are generally accepted as the standard chemotherapy for advanced gastric cancer $[12,13]$. In particular, the Japanese Randomized Control Trial (RCT) (SPIRITS trial) showed that S-1 (an oral fluoropyrimidine derivative) plus CDDP is the standard regimen for first-line treatment of advanced gastric 
cancer cases with an unresectable or recurrent tumor [8]. However, there is no established standard regimen specific for peritoneal metastasis, except for a few early-phase clinical trials. For example, systemic sequential methotrexate and 5-FU therapy has been reported to decrease malignant ascites in gastric cancer patients, although the 1-year survival rate was $16.2 \%$ [14]. Another trial indicated the efficacy of systemic modified FOLFOX4, which yielded a 1-year survival rate of $27.2 \%$ in gastric cancer patients with malignant ascites [15]. Because of unsatisfactory results in these phase II clinical trials with systemic chemotherapy, no regimen is currently accepted as the standard chemotherapy for peritoneal dissemination.

Regarding intraperitoneal chemotherapy, it has been reported that intraperitoneal administration of MMC or CDDP resulted in no significant clinical effects against peritoneal metastasis of gastric cancer [3]. The likely reason for this failure is that both MMC and CDDP are hydrophilic, low-weight compounds that are rapidly absorbed through the peritoneal mesothelial layer, leading to pharmacokinetic insufficiency in the peritoneal cavity or poor sensitivity of cancer cells to the drug.

As for alternative options for gastric cancer with peritoneal dissemination, it has been reported that cytoreductive surgery (CRS) combined with hyperthermic intraperitoneal chemotherapy (HIPEC) has the potential to control peritoneal disease in gastric cancer, as it led to median survival times and 1-year survival rates of 9.2-11.5 months and 35.5-48.1\%, respectively [16-19]. However, no RCT has confirmed the clinical efficacy of CRS plus HIPEC for gastric cancer, and this procedure is associated with high mortality and morbidity [20]. The clinical significance of CRS plus HIPEC for gastric cancer is thus currently debatable and under investigation.

\section{Intraperitoneal chemotherapy in Japan: phase II clinical trials}

As described above, S-1 is a key drug for gastric cancer, yielding response rates of $27-31 \%$ and median survival times of 10.5-11.4 months for advanced gastric cancer patients $[8,9]$. Moreover, intravenous paclitaxel with triweekly regimens in phase II studies have shown response rates of $20-28 \%$ and median survival times of 7.8-11 months for advanced gastric cancer in the first-line and second-line settings [21-23]. Thus, both drugs highly sensitively target diffuse-type adenocarcinoma and are able to efficiently pass into the peritoneal cavity [23-26], indicating that a combination of S-1 with intravenous paclitaxel has the potential to exert a synergistic effect for peritoneal dissemination. In fact, in advanced gastric cancer patients with distant metastasis or recurrence, several clinical trials with $\mathrm{S}-1$ plus intravenous paclitaxel have already reported on the safety and efficacy of this combination, highlighting its promising clinical effects [27-29].

Furthermore, since taxanes such as paclitaxel or docetaxel are hydrophobic, high-weight compounds [30], taxanes are absorbed slowly through the lymphatic system after intraperitoneal administration due to their high molecular weights and fat solubilities, leading to high drug concentrations in the peritoneal cavity [31]. Therefore, a combination of systemic chemotherapy with intraperitoneal taxanes is expected to be the most powerful and effective treatment for peritoneal metastasis. In fact, several phase II clinical trials using systemic chemotherapy with intraperitoneal taxanes have reported promising results (Table 1 [32-36]), although the trial reported by Imano et al. [34] consisted of a single intraperitoneal dose of paclitaxel. Among these trials, a phase II clinical trial with a combination of S-1 plus intravenous and intraperitoneal paclitaxel was conducted for patients with unresectable or recurrent gastric cancer limited to the peritoneum and/or cancer cells in peritoneal cytology [32]. As a result, the combination of intraperitoneal and intravenous paclitaxel with S-1 permitted a median survival time of 23.6 months and a 1-year overall survival rate of $78 \%$ in a phase II study [32]. Peritoneal cytology turned negative for $86 \%$ of the patients. In an updated report, gastrectomy was safely performed on 52 patients after the peritoneal metastasis had disappeared [37].

Regarding safety and feasibility in the phase II study [32], grade $3 / 4$ hematological and nonhematological adverse effects were seen in 40 and $15 \%$ of the patients, respectively. The grade $3 / 4$ toxic effects included neutropenia (38\%), leukopenia (18\%), and anemia (10\%). Obstruction of the intraperitoneal catheter was observed in only 1 of 40 patients. There were no treatment-related deaths. These results strongly suggest that the combination chemotherapy of S-1 plus intravenous and intraperitoneal paclitaxel is safe and feasible and has promising clinical effects. Therefore, a phase III RCT (PHOENIX-GC trial) with this regimen was planned.

\section{PHOENIX-GC trial in Japan (clinical trial information: UMIN000005930)}

A multicenter phase III study, the PHOENIX-GC trial, evaluated the clinical efficacy of intraperitoneal paclitaxel plus systemic S-1 and paclitaxel compared to standard systemic chemotherapy (S-1 + CDDP), in which the primary endpoint was overall survival and the secondary endpoints were response rate and safety. Patients were randomly assigned in a 2:1 ratio into an intraperitoneal chemotherapy group (intraperitoneal paclitaxel $20 \mathrm{mg} / \mathrm{m}^{2}$, 
Table 1 Phase II clinical trials with intraperitoneal taxanes for gastric cancer patients with peritoneal dissemination

\begin{tabular}{lllllc}
\hline Authors & Number of patients & Intraperitoneal taxane & MST (months) & 1-year OS (\%) & 2-year OS (\%) \\
\hline Ishigami et al. [32] & 40 & PTX $\left(20 \mathrm{mg} / \mathrm{m}^{2}\right)$ & 22.6 & 78 & 76 \\
Fujiwara et al. [33] & 18 & DOC $\left(40-60 \mathrm{mg} / \mathrm{m}^{2}\right)$ & 24.6 & 69 & 46 \\
Imano et al. [34] & 35 & PTX $\left(80 \mathrm{mg} / \mathrm{m}^{2}\right)$ & 21.3 & 77 & 45 \\
Yamaguchi et al. [35] & 35 & PTX $\left(20 \mathrm{mg} / \mathrm{m}^{2}\right)$ & 17.6 & 70 & 33 \\
Fushida et al. [36] & 27 & DOC $\left(35-50 \mathrm{mg} / \mathrm{m}^{2}\right)$ & 16.2 & \\
\hline
\end{tabular}

$M S T$ median survival time, $O S$ overall survival, $D O C$ docetaxel, $P T X$ paclitaxel

intravenous paclitaxel $50 \mathrm{mg} / \mathrm{m}^{2}$ on days 1 and 8 , and $\mathrm{S}-1$ $80 \mathrm{mg} / \mathrm{m}^{2} /$ day on days $1-14$, q3 weeks) or a standard chemotherapy group (S-1 + CDDP; CDDP $60 \mathrm{mg} / \mathrm{m}^{2}$ on day 8 , and S-1 $80 \mathrm{mg} / \mathrm{m}^{2} /$ day on days $1-21$, q5 weeks). According to results presented at the 2016 ASCO, 183 patients were enrolled and 164 patients were included in the efficacy analysis. The background characteristics of the patients in the two groups were similar except for the degree of ascites. The median overall survival for the intraperitoneal group and that for the standard chemotherapy group were 17.7 and 15.2 months, respectively (stratified log-rank test, $p=0.080$; hazard ratio $0.72,95 \%$ confidence interval $0.49-1.04, p=0.081$ ). The overall response rate was $53 \%$ in the peritoneal chemotherapy group, whereas it was $60 \%$ in the standard chemotherapy group $(p=1.0)$. Both regimens were well tolerated and there were no treatment-related death. In an initial report of the PHOENIX-GC trial, the study did not statistically demonstrate a superiority of the intraperitoneal chemotherapy regimen over the systemic standard chemotherapy. The sensitivity analysis, which took into account the imbalance in ascites between the groups, suggested that intraperitoneal paclitaxel is clinically effective for treating gastric cancer with peritoneal metastasis.

\section{Intraperitoneal chemotherapy in Singapore}

Since S-1 is not yet widely available in Singapore, the combination of capecitabine with oxaliplatin (XELOX) is currently one of the most common chemotherapy regimens for patients with advanced gastric cancer there. Therefore, it is desirable to determine if the combination of intraperitoneal paclitaxel with XELOX has favorable clinical efficacy for gastric cancer with peritoneal dissemination. For this purpose, we designed a clinical trial, a single-arm phase II trial, that evaluated the efficacy and tolerability of intraperitoneal paclitaxel with oxaliplatin and capecitabine in advanced gastric cancer patients with peritoneal metastasis and/or cancer cells on peritoneal

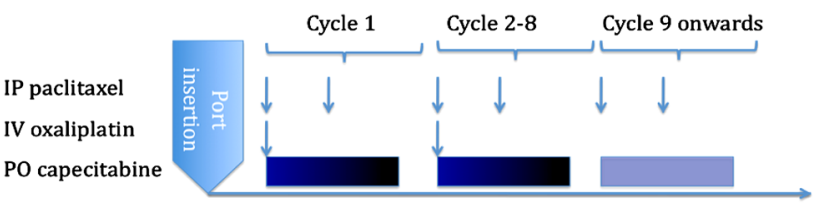

Fig. 2 Protocol of the phase II clinical trial of intraperitoneal chemotherapy for gastric cancer in Singapore. Intraperitoneal (IP) paclitaxel with intravenous (IV) oxaliplatin and capecitabine was administered to advanced gastric cancer patients with peritoneal metastasis and/or cancer cells on peritoneal cytology. One cycle constituted intraperitoneal paclitaxel being administered at $40 \mathrm{mg} / \mathrm{m}^{2}$ on days 1 and 8 , oxaliplatin given at $100 \mathrm{mg} / \mathrm{m}^{2} /$ day for day 1 , and oral capecitabine administered at $1000 \mathrm{mg} / \mathrm{m}^{2}$ twice daily for 14 days continuously, followed by 1 week of rest. This regimen was repeated for 8 cycles

cytology (Fig. 2). One cycle constituted intraperitoneal paclitaxel being administered at $40 \mathrm{mg} / \mathrm{m}^{2}$ on days 1 and 8 , oxaliplatin given at $100 \mathrm{mg} / \mathrm{m}^{2} /$ day for day 1 , and oral capecitabine administered at $1000 \mathrm{mg} / \mathrm{m}^{2}$ twice daily for 14 days continuously, followed by 1 week of rest. This regimen was repeated for 8 cycles. The primary endpoint was the 1-year overall survival rate and the secondary endpoints were overall response rate, negative peritoneal cytology rate, and safety. The dose of intraperitoneal paclitaxel was derived from a phase I study of intraperitoneal paclitaxel with oxaliplatin and S-1 [38]. Both S-1 and capecitabine are orally available fluoropyrimidines that have been combined with oxaliplatin and found to be equally effective and well tolerated in patients with advanced gastric cancer [39]. The reported 1-year survival for advanced gastric cancer patients was $42 \%$ in patients treated with standard systemic chemotherapy in the AVAGAST study [40], and $78 \%$ in the study combining intraperitoneal paclitaxel with standard chemotherapy [32]. If the new treatment achieved a 1-year survival of $70 \%$ then further development and trials were considered worthwhile, but if the cure rate was no better than the existing treatment (0.42) then further development was to be abandoned. With $A=0.05$ and power $=0.8$, using Fleming's model, a sample size of 16 patients was required. A total of 20 subjects were recruited for the study 
over a two-year period in the initial design. In short, the study had the power to detect a 1-year survival of $70 \%$ with 22 patients. The study was then completed and reached the survival endpoint. However, there was 1 case of G5 neutropenic sepsis and 1 G5 cerebral vascular accident which may not be treatment-related. Therefore, the safety committee recommended increasing the recruitment to 40 subjects to better assess the tolerability of this regimen.

In the analysis of the first 22 cases recruited, the 1-year overall survival rate was $72 \%$ and the median survival time was 18.8 months, which are comparable to or less effective than those observed in the phase II clinical trial of intraperitoneal paclitaxel, intravenous paclitaxel, and S-1 in Japan [32] (78 \% and 22.5 months, respectively). The final results for all 40 patients are awaited.

\section{Conclusion}

In the initial report of the Phoenix-GC trial, the trial did not meet the primary endpoint to show the clinical significance of intraperitoneal paclitaxel in comparison to systemic chemotherapy. More detailed analysis of the Phoenix-GC trial is needed to draw a solid conclusion regarding the clinical significance of intraperitoneal chemotherapy. Moreover, the final results from the Singapore trial of intraperitoneal paclitaxel with XELOX are also awaited.

\section{Compliance with ethical standards}

Ethical statements All procedures in this study were in accordance with the ethical standards of the responsible committee on human experimentation (institutional and national) and with the Helsinki Declaration of 1964.

Conflict of interest The authors have no conflicting financial interest regarding the present study.

\section{References}

1. Ferlay J, Soerjomataram I, Dikshit R, Eser S, Mathers C, Rebelo $\mathrm{M}$, et al. Cancer incidence and mortality worldwide: sources, methods and major patterns in GLOBOCAN 2012. Int J Cancer. 2015;136:E359-86.

2. Kuramoto M, Shimada S, Ikeshima S, Matsuo A, Yagi Y, Matsuda $\mathrm{M}$, et al. Extensive intraoperative peritoneal lavage as a standard prophylactic strategy for peritoneal recurrence in patients with gastric carcinoma. Ann Surg. 2009;250:242-6.

3. Sautner T, Hofbauer F, Depisch D, Schiessel R, Jakesz R. Adjuvant intraperitoneal cisplatin chemotherapy does not improve long-term survival after surgery for advanced gastric cancer. J Clin Oncol. 1994;12:970-4.

4. Francis P, Rowinsky E, Schneider J, Hakes T, Hoskins W, Markman M. Phase I feasibility and pharmacologic study of weekly intraperitoneal paclitaxel: a gynecologic oncology group pilot study. J Clin Oncol. 1995;13:2961-7.
5. Armstrong DK, Bundy B, Wenzel L, Huang HQ, Baergen R, Lele $\mathrm{S}$, et al. Intraperitoneal cisplatin and paclitaxel in ovarian cancer. N Engl J Med. 2006;354:34-43.

6. Lim GH, Chow KY, Lee HP. Singapore cancer trends in the last decade. Singap Med J. 2012;53:3-9.

7. Chong CS, Ng CW, Shabbir A, Kono K, So JB. Computed tomography of the thorax for gastric cancer staging: is it necessary? Scand J Surg. 2015;104:244-7.

8. Koizumi W, Narahara H, Hara T, Takagane A, Akiya T, Takagi $\mathrm{M}$, et al. S-1 plus cisplatin versus S-1 alone for first-line treatment of advanced gastric cancer (SPIRITS trial): a phase III trial. Lancet Oncol. 2008;9:215-21.

9. Boku N. Gastrointestinal Oncology Study Group of Japan Clinical Oncology G. Chemotherapy for metastatic disease: review from JCOG trials. Int J Clin Oncol. 2008;13:196-200.

10. Sugarbaker PH, Yonemura Y. Clinical pathway for the management of resectable gastric cancer with peritoneal seeding: best palliation with a ray of hope for cure. Oncology. 2000;58:96-107.

11. Fujimoto S, Takahashi M, Kobayashi K, Kure M, Mutou T, Masaoka $\mathrm{H}$, et al. Relation between clinical and histologic outcome of intraperitoneal hyperthermic perfusion for patients with gastric cancer and peritoneal metastasis. Oncology. 1993;50:338-43

12. Ajani JA. Standard chemotherapy for gastric carcinoma: is it a myth? J Clin Oncol. 2000;18:4001-3.

13. Ohtsu A, Yoshida S, Saijo N. Disparities in gastric cancer chemotherapy between the East and West. J Clin Oncol. 2006;24:2188-96.

14. Yamao T, Shimada Y, Shirao K, Ohtsu A, Ikeda N, Hyodo I, et al. Phase II study of sequential methotrexate and 5-fluorouracil chemotherapy against peritoneally disseminated gastric cancer with malignant ascites: a report from the Gastrointestinal Oncology Study Group of the Japan Clinical Oncology Group, JCOG 9603 Trial. Jpn J Clin Oncol. 2004;34:316-22.

15. Oh SY, Kwon HC, Lee S, Lee DM, Yoo HS, Kim SH, et al. A phase II study of oxaliplatin with low-dose leucovorin and bolus and continuous infusion 5-fluorouracil (modified FOLFOX-4) for gastric cancer patients with malignant ascites. Jpn J Clin Oncol. 2007;37:930-5.

16. Glehen O, Schreiber V, Cotte E, Sayag-Beaujard AC, Osinsky D, Freyer G, et al. Cytoreductive surgery and intraperitoneal chemohyperthermia for peritoneal carcinomatosis arising from gastric cancer. Arch Surg. 2004;139:20-6.

17. Yonemura Y, Kawamura T, Bandou E, Takahashi S, Sawa T, Matsuki N. Treatment of peritoneal dissemination from gastric cancer by peritonectomy and chemohyperthermic peritoneal perfusion. Br J Surg. 2005;92:370-5.

18. Yang XJ, Huang CQ, Suo T, Mei LJ, Yang GL, Cheng FL, et al. Cytoreductive surgery and hyperthermic intraperitoneal chemotherapy improves survival of patients with peritoneal carcinomatosis from gastric cancer: final results of a phase III randomized clinical trial. Ann Surg Oncol. 2011;18:1575-81.

19. Glehen O, Gilly FN, Arvieux C, Cotte E, Boutitie F, Mansvelt B, et al. Peritoneal carcinomatosis from gastric cancer: a multi-institutional study of 159 patients treated by cytoreductive surgery combined with perioperative intraperitoneal chemotherapy. Ann Surg Oncol. 2010;17:2370-7.

20. Gill RS, Al-Adra DP, Nagendran J, Campbell S, Shi X, Haase E, et al. Treatment of gastric cancer with peritoneal carcinomatosis by cytoreductive surgery and HIPEC: a systematic review of survival, mortality, and morbidity. J Surg Oncol. 2011;104:692-8.

21. Ajani JA, Fairweather J, Dumas P, Patt YZ, Pazdur R, Mansfield PF. Phase II study of taxol in patients with advanced gastric carcinoma. Cancer J Sci Am. 1998;4:269-74. 
22. Ohtsu A, Boku N, Tamura F, Muro K, Shimada Y, Saigenji K, et al. An early phase II study of a 3-hour infusion of paclitaxel for advanced gastric cancer. Am J Clin Oncol. 1998;21:416-9.

23. Yamada Y, Shirao K, Ohtsu A, Boku N, Hyodo I, Saitoh H, et al. Phase II trial of paclitaxel by three-hour infusion for advanced gastric cancer with short premedication for prophylaxis against paclitaxel-associated hypersensitivity reactions. Ann Oncol. 2001;12:1133-7.

24. Koizumi W, Kurihara M, Nakano S, Hasegawa K. Phase II study of S-1, a novel oral derivative of 5-fluorouracil, in advanced gastric cancer. For the S-1 Cooperative Gastric Cancer Study Group. Oncology. 2000;58:191-7.

25. Mori T, Fujiwara Y, Yano M, Tamura S, Yasuda T, Takiguchi S, et al. Prevention of peritoneal metastasis of human gastric cancer cells in nude mice by $\mathrm{S}-1$, a novel oral derivative of 5-fluorouracil. Oncology. 2003;64:176-82.

26. Kobayashi M, Sakamoto J, Namikawa T, Okamoto K, Okabayashi T, Ichikawa K, et al. Pharmacokinetic study of paclitaxel in malignant ascites from advanced gastric cancer patients. World J Gastroenterol. 2006;12:1412-5.

27. Fujitani K, Narahara H, Takiuchi H, Tsujinaka T, Satomi E, Gotoh M, et al. Phase I and pharmacokinetic study of S-1 combined with weekly paclitaxel in patients with advanced gastric cancer. Oncology. 2005;69:414-20.

28. Mochiki E, Ohno T, Kamiyama Y, Aihara R, Haga N, Ojima H, et al. Phase I/II study of S-1 combined with paclitaxel in patients with unresectable and/or recurrent advanced gastric cancer. Br J Cancer. 2006;95:1642-7.

29. Narahara H, Fujitani K, Takiuchi H, Sugimoto N, Inoue K, Uedo $\mathrm{N}$, et al. Phase II study of a combination of S-1 and paclitaxel in patients with unresectable or metastatic gastric cancer. Oncology. 2008;74:37-41.

30. Flessner MF, Fenstermacher JD, Blasberg RG, Dedrick RL. Peritoneal absorption of macromolecules studied by quantitative autoradiography. Am J Physiol. 1985;248:H26-32.

31. Ishigami H, Kitayama J, Kaisaki S, Yamaguchi H, Yamashita H, Emoto S, et al. Phase I study of biweekly intravenous paclitaxel plus intraperitoneal cisplatin and paclitaxel for gastric cancer with peritoneal metastasis. Oncology. 2010;79:269-72.
32. Ishigami H, Kitayama J, Kaisaki S, Hidemura A, Kato M, Otani $\mathrm{K}$, et al. Phase II study of weekly intravenous and intraperitoneal paclitaxel combined with S-1 for advanced gastric cancer with peritoneal metastasis. Ann Oncol. 2010;2:67-70.

33. Fujiwara Y, Takiguchi S, Nakajima K, Miyata H, Yamasaki M, Kurokawa Y, et al. Intraperitoneal docetaxel combined with S-1 for advanced gastric cancer with peritoneal dissemination. J Surg Oncol. 2012;105:38-42.

34. Imano M, Peng YF, Itoh T, Nishikawa M, Satou T, Yasuda A, et al. A preliminary study of single intraperitoneal administration of paclitaxel followed by sequential systemic chemotherapy with S-1 plus paclitaxel for advanced gastric cancer with peritoneal metastasis. Anticancer Res. 2012;32:4071-5.

35. Yamaguchi H, Kitayama J, Ishigami H, Emoto S, Yamashita H, Watanabe T. A phase 2 trial of intravenous and intraperitoneal paclitaxel combined with S-1 for treatment of gastric cancer with macroscopic peritoneal metastasis. Cancer. 2013;119:3354-8.

36. Fushida S, Kinoshita J, Kaji M, Hirono Y, Goda F, Yagi Y, et al. Phase I/II study of intraperitoneal docetaxel plus S-1 for the gastric cancer patients with peritoneal carcinomatosis. Cancer Chemother Pharmacol. 2013;71:1265-72.

37. Kitayama J, Ishigami $\mathrm{H}$, Yamaguchi $\mathrm{H}$, Yamashita $\mathrm{H}$, Emoto $\mathrm{S}$, Kaisaki S, et al. Salvage gastrectomy after intravenous and intraperitoneal paclitaxel (PTX) administration with oral S-1 for peritoneal dissemination of advanced gastric cancer with malignant ascites. Ann Surg Oncol. 2014;21:539-46.

38. Ishigami H, Kaisaki S, Yamaguchi H, et al. Phase I study of weekly intraperitoneal paclitaxel combined with S-1 and oxaliplatin for gastric cancer with peritoneal metastasis. J Clin Oncol. 2012;30(suppl 4):146.

39. Kim GM, Jeung HC, Rha SY, Kim HS, Jung I, Nam BH, et al. A randomized phase II trial of S-1-oxaliplatin versus capecitabineoxaliplatin in advanced gastric cancer. Eur $\mathrm{J}$ Cancer. 2012;48:518-26.

40. Ohtsu A, Shah MA, Van Cutsem E, Rha SY, Sawaki A, Park SR, et al. Bevacizumab in combination with chemotherapy as firstline therapy in advanced gastric cancer: a randomized, doubleblind, placebo-controlled phase III study. J Clin Oncol. 2011;29:3968-76. 